(C) 2000 International Press

Adv. Theor. Math. Phys. 4 (2000) 249-269

\title{
Matrix theory star products from the Born-Infeld action
}

Lorenzo Cornalba and Ricardo Schiappa

\author{
Institut des Hautes Études Scientifiques \\ Le Bois-Marie, Bures-Sur-Yvette, 91440 France \\ cornalba@ihes.fr \\ Department of Physics \\ Harvard University \\ Cambridge, MA 02138, U.S.A. \\ ricardo@lorentz.harvard.edu
}

\begin{abstract}
We conjecture that the Sen-Seiberg limit of the Type IIA D2-brane action in a flat spacetime background can be resummed, at all orders in $\alpha^{\prime}$, to define an associative star product on the membrane. This star product can be independently
\end{abstract}

e-print archive: http://xxx.lanl.gov/hep-th/9907211 
constrained from the equivalent Matrix theory description of the corresponding $M 2$-brane, by carefully analyzing the known BPS conditions. Higher derivative corrections to the Born-Infeld action on the IIA side are reinterpreted, after the Sen-Seiberg limit, as higher derivative corrections to a field theory on the membrane, which itself can be resummed to yield the known Matrix theory quantum mechanics action. Conversely, given the star product on the membrane as a formal power series in $\alpha^{\prime}$, one can constrain the higher derivative corrections to the BornInfeld action, in the Sen-Seiberg limit. This claim is explicitly verified to first order. Finally, we also comment on the possible application of this method to the derivation of the Matrix theory action for membranes in a curved background.

\section{Introduction and Discussion}

It is by now clear that string theory as it emerged in the eighties is but a fraction of the full story. The five known superstring theories as well as the low-energy 11-dimensional supergravity are now known to be related through a web of dualities [1], and it is believed that all these theories are simply different limits of an underlying 11-dimensional quantum theory known as $M$-theory, whose fundamental degrees of freedom are as yet unknown. Throughout these string theories we have $D$-branes of various dimensions [2], playing in certain regimes a role as fundamental as that of the basic string. These $D$-branes have a precise description within string theory, their low-energy dynamics being dictated by the Born-Infeld action [3] which can be obtained using boundary conformal field theory techniques. $M$-theory itself contains M2-branes and $M 5$-branes, which themselves can be related to the branes in string theories [1].

The problem of understanding the structure of string theory is directly related to a full understanding of what is the fundamental nature of spacetime. At large distances the structure of the spacetime manifold is well described by Riemannian geometry. On the other hand, at small distances - i.e., much shorter than the string scale $\ell_{s}$ - the Riemannian description of spacetime breaks down. In fact, there is as yet no known intrinsic and covariant description of the underlying 
geometry and fundamental degrees of freedom. Despite this lack of understanding of the small distance structure, one can use $D$-branes in order to probe physics at distances smaller than the string length [4].

In this work we shall focus our attention on the description of the $M$-theory $M 2$-brane. Let us first recall that $M$-theory compactified on a circle is described by Type IIA string theory at finite string coupling [1]. Moreover, the (unwrapped) M2-brane corresponds, in the Type IIA description, to the Dirichlet membrane. It is by now a well known conjecture that $M$-theory compactified on a light-like circle admits a non-perturbative description in terms of the degrees of freedom of a collection of $D 0$-branes $[5,6,7,8]$. Moreover, these $D 0$-branes are exactly described by the $(0+1)$-dimensional reduction of 10 -dimensional $U(N)$ super Yang-Mills theory. A precise understanding of this Matrix theory conjecture is obtained by starting with $M$-theory, now compactified on a space-like circle, and considering the sector with $N$ units of momenta in the compact direction. From a IIA point of view this corresponds to the sector of the theory with $N D 0$-branes. One then uses the Poincaré invariance of the underlying $M$-theory in order to relate the space-like compactified theory to the theory compactified on the light-cone. This is achieved by both sending the space-like compactification radius to zero and, at the same time, by rescaling the 11-dimensional Planck length. Such a prescription is known as the Sen-Seiberg limit $[9,10]$.

Membranes exist in both Type IIA string theory and in $M$-theory. In particular, one should be able to describe $M 2-$ branes as specific states within Matrix theory. On the other hand, D2-branes can be described within Type IIA string theory in terms of the geometry of a world-volume manifold embedded in spacetime, whose dynamics are governed at low energies by the Born-Infeld action and in general, at weak coupling, by the full $\alpha^{\prime}$ expansion of the boundary state conformal field theory [3]. Naively one would assume that these two descriptions should be essentially the same - i.e., they should coincide in the Sen-Seiberg limit. On the other hand, a closer look shows that these descriptions are actually valid in seemingly different regimes. In fact, Matrix theory probes the dynamics of the $D 0$-branes at the Planckian length which, in the Sen-Seiberg limit, is much smaller than the string length. The $\alpha^{\prime}$ expansion is, on the other hand, a large distance expansion, therefore valid in the opposite regime. Still, we shall conjecture in this work that one can learn about one description from the other. 
In particular, the exact knowledge of the Matrix theory action can be used to conjecture the Sen-Seiberg limit of the action describing the D2-brane to all orders in $\alpha^{\prime}$ - i.e., Born-Infeld plus higher derivative corrections. Conversely, one could proceed the other way around and use extensions of known results about $D 2$-branes propagating in nonflat spacetime [11] in order to start tackling the problem of describing Matrix theory in curved backgrounds. Indeed one could use conformal field theory calculations in order to determine the $\alpha^{\prime}$ corrections to the Born-Infeld action in a curved background. Then, via the prescription described in this paper, one could take the Sen-Seiberg limit and obtain the first corrections to Matrix theory in this curved background $[12,13,14,15,16]$.

Let us now be a bit more specific. In order to carry out the above program, we shall start by considering static BPS membranes in Matrix theory. At the classical level, in flat background spacetime, these membranes are described by holomorphic curves. Using the work of Cornalba and Taylor $[17,18]$, one can represent any holomorphically embedded curve via matrices - i.e., we can construct its matrix representation. This construction is accomplished by starting with a given holomorphic embedding and then associating functions on the membrane to matrices, so that matrix multiplication is represented by an associative star product on the space of functions. This star product is clearly non-commutative and, moreover, it is given by a formal power expansion in $\alpha^{\prime}=\ell_{P}^{3} / R$, which at each order is given by a local derivative bilinear of the functions living on the membrane. The product starts as simple function multiplication, and is constrained at higher orders by the following three conditions. First, the star commutator of functions starts at order $\alpha^{\prime}$ with the Poisson bracket, where the symplectic form is determined by the area element of the embedded curve. Secondly, and as previously stated, the product should be associative. Finally, the coordinate functions which represent at the Matrix level the static curve, should satisfy the BPS condition which follows from the known Matrix theory action. As described in [17] these three conditions can be iteratively solved (algebraically) in powers of $\alpha^{\prime}$, if one considers the specific case of holomorphic quantization for the BPS membranes.

Having described the static BPS branes, we now proceed by considering fluctuations about these solutions. Fluctuations can again be represented dually either as matrices or as functions on the brane. In 
particular, if we consider the fluctuating coordinates as functions and we re-write the Matrix theory action in terms of the star product, one immediately gets an action in terms of higher derivatives of these coordinates functions and in powers of $\alpha^{\prime}$. It is then very natural to conjecture that this expansion is nothing but the Sen-Seiberg limit of the full $\alpha^{\prime}$ expansion describing the $D 2$-brane.

What we do in this paper is, first of all, to describe in detail the correct Sen-Seiberg limit of the D2-brane action. We then apply this procedure to the Born-Infeld action and show that, in the limit, it reproduces exactly the light-cone gauge fixed Nambu-Goto $M 2$-brane action in 11-dimensions. This is, as expected, the first term in the previously described expansion of the Matrix theory action as given in terms of the star product. Finally, we describe how one should proceed in order to test the conjecture at higher orders in $\alpha^{\prime}$, and moreover how one can infer higher derivative terms in the $D 2$-brane action from a given star product.

The picture that emerges from this conjecture is the following. Each derivative correction to the Born-Infeld action translates into a correction to the effective field theory on the $M 2$-brane. The sum of all these derivative corrections on the $D 2$-brane action translate to an infinite sum of corrections to the field theory on the $M 2$-brane, in such a way that, in the Sen-Seiberg limit, they can be re-organized into a star product. This reduces the non-renormalizable field theory on the $\mathrm{M2}_{2}$ brane to a matrix quantum mechanics. Moreover, this star product can be independently constrained by analyzing the BPS condition arising from Matrix theory.

Throughout the paper; we shall only discuss the bosonic part of the actions which we consider. Let us recall though that we are always analyzing supersymmetric theories, and therefore all the actions have a SUSY extension involving the dynamics of the corresponding fermionic fields. The main point of the present paper is most simply described within the bosonic theory, and we therefore omit the fermionic parts of the actions.

While this paper was being typed, we learned about other work along similar lines [19]. 


\section{The Sen-Seiberg Limit of the Born-Infeld Action}

Our ultimate goal is to show how one can relate the action for an $M$-theory membrane to the action describing a $D 2$-brane in Type IIA string theory. To this end, we have to analyze the Sen-Seiberg limit and precisely understand how it relates states in light-cone compactified $M$ theory to states in Type IIA theory. In this section in particular, we shall consider the motion of an $\mathrm{M} 2$-brane as described by the NambuGoto action and we will show that the dynamics can be determined from the Born-Infeld action describing the $D 2$-brane through a precise understanding of its Sen-Seiberg limit.

We start, in order to introduce notation ${ }^{1}$, by reviewing the standard light-cone description of $M$-theory in a flat background. Transverse coordinates will be denoted by $X^{i}, i=1, \ldots, 9$, while light-cone ones by $X^{ \pm}=2^{-1 / 2}\left(X^{0} \pm X^{10}\right)$. Also, $\ell_{P}$ will denote the Planck length, and $R$ the light-like compactification radius in the $X^{-}$direction. To describe the motion of an $M 2$-brane (we shall always consider $M 2-$ branes which do not wrap the compact direction), we take the worldvolume of the membrane to be the product space $\mathbf{R} \times \Sigma$, where $\Sigma$ is a two dimensional surface with the topology of the brane. Coordinates on the world-volume are $\tau$ and $\sigma^{a}$ (with $a=1,2$ ) or, collectively, $\xi^{\alpha}$ (with $\alpha=0,1,2$ ). The motion of the surface is described by coordinate functions $X^{\mu}: \mathbf{R} \times \Sigma \rightarrow \mathbf{R}^{11}$ on the world-volume, and the correct dynamics is determined, at low energy, by the minima of the membrane Nambu-Goto action,

$$
S=-T \int d^{3} \xi \sqrt{-\operatorname{det} g_{\alpha \beta}}
$$

where $g_{\alpha \beta}=\partial_{\alpha} X^{\mu} \partial_{\beta} X_{\mu}$ is the induced metric, and the tension $T$ of the brane is given by

$$
T=\frac{1}{(2 \pi)^{2} \ell_{P}^{3}} .
$$

${ }^{1}$ We will use the following index conventions. Indices in $M$-theory spacetime will be $\mu, \nu, \ldots$, and on the brane world-volume will be $\alpha, \beta, \ldots$ Spatial indices on the brane world-volume will be $a, b, \ldots$, and spatial directions in IIA (or equivalently transverse directions in $M$-theory) will be $i, j, \ldots$. 
In order to simplify the highly non-polynomial Nambu-Goto action, we can use the diffeomorphism invariance on the brane world-volume. In fact, it is well known $[20,21,22]$ that the propagation of the $M 2$-brane is most conveniently described in the light-front gauge, where the independent degrees of freedom are the transverse embedding coordinates $X^{i}$ and the remaining light-front coordinates $X^{ \pm}$are given by the gauge constraints $X^{+}=\tau$ and $\partial_{a} X^{-}=\dot{X}^{i} \partial_{a} X^{i}$. The dynamics of the system is then governed by the Lagrangian $[20,21]$

$$
L=\frac{T}{\sqrt{2}} \int d^{2} \sigma Q\left(\frac{1}{2} \dot{X}^{i} \dot{X}^{i}-\frac{1}{2}\left\{X^{i}, X^{j}\right\}^{2}\right),
$$

where $d^{2} \sigma Q$ is a fixed volume form on the manifold $\Sigma$ describing the brane and

$$
\{A, B\}=\frac{1}{Q} \varepsilon^{a b} \partial_{a} A \partial_{b} B
$$

is the Poisson bracket corresponding to the volume form. The overall normalization of the action has been chosen so that, when considering static solutions of the equations of motion, the area element $Q d^{2} \sigma$ corresponds to the area element of the brane given by the embedding in the transverse directions. More precisely, if

$$
h_{a b}=\partial_{a} X^{i} \partial_{b} X^{i}
$$

then, for solutions such that $X^{ \pm}=\tau$ and $\dot{X}^{i}=0$, we have that $Q=$ $\sqrt{\operatorname{det} h_{a b}}$. More generally

$$
Q=\sqrt{\frac{\operatorname{det} h_{a b}}{\dot{X}^{-}-\frac{1}{2} \dot{X}^{i} \dot{X}^{i}}} .
$$

Finally, let us recall that, in order to solve the gauge constraint $\partial_{a} X^{-}=$ $\dot{X}^{i} \partial_{a} X^{i}$, we clearly have to restrict our attention to solutions satisfying the constraint

$$
\left\{X^{i}, \dot{X}^{i}\right\}=0 .
$$

We now wish to rederive the above description of the $M 2$-brane, this time starting from the known duality between $M$-theory and Type IIA [1]. First, let us recall $[6,8,9,10]$ that $M$-theory compactified on 
the light-like circle $X^{-} \sim X^{-}+2 \pi R$ can be described as the limit of $M$-theory compactified on a space-like circle of radius $\widetilde{R}$, where we take the Sen-Seiberg limit $\widetilde{R} \rightarrow 0$. Moreover, in order to match energy levels $[9,10]$, we need to consider $M$-theory with a new Planck length $\widetilde{\ell_{P}}$, where we keep the ratio $R / \ell_{P}^{2}=\widetilde{R} /{\widetilde{\ell_{P}}}^{2}$ constant. In the following, we shall refer to the space-like compactified theory as $\widetilde{M}$. In order to describe the above limit in detail, we introduce a dimensionless parameter $\eta \rightarrow 0$, and we then take

$$
\widetilde{\ell_{P}}=\eta^{2} \ell_{P}, \quad \widetilde{R}=\eta^{4} R .
$$

If we denote by $\widetilde{X}^{\mu}$ the space-time coordinates in the $\widetilde{M}$-theory, we have the relations

$$
\begin{aligned}
\tilde{X}^{0} & =X^{+} \\
\widetilde{X}^{10} & \sim \widetilde{X}^{10}+2 \pi \widetilde{R} \\
\widetilde{X}^{i} & =\eta^{2} X^{i}
\end{aligned}
$$

Consider, in particular, a state in the theory $M$ with momentum $P^{\mu}$. The momentum $P^{+}$corresponding to the compact direction $X^{-}$will be quantized as

$$
P^{+}=\frac{N}{R} \text {. }
$$

Moreover, the corresponding state in the $\widetilde{M}$-theory will have momentum $\widetilde{P}^{\mu}$, where

$$
\begin{aligned}
\widetilde{P}^{0} & =N / \widetilde{R}+P^{+} \\
\widetilde{P}^{10} & =N / \widetilde{R} \\
\widetilde{P}^{i} & =\eta^{-2} P^{i}
\end{aligned}
$$

Recall that $M$-theory compactified on a space-like circle is given by Type IIA string theory at finite coupling [1]. Therefore, in order to describe the $\widetilde{M}$ - theory with $N$ units of momentum in the compact direction, one needs to consider Type IIA with $N$ units of $D 0$-brane charge and with the following string length and coupling

$$
\widetilde{\ell_{s}}=\eta \ell_{s} \quad, \quad \widetilde{g_{s}}=\eta^{3} g_{s}
$$


where we have introduced the constants

$$
\ell_{s}^{2}=\frac{\ell_{P}^{3}}{R} \quad, \quad g_{s}=\left(\frac{R}{\ell_{P}}\right)^{3 / 2},
$$

related to the original light-like compactified $M$-theory. Finally, we introduce the constants

$$
\alpha^{\prime}=\ell_{s}^{2} \quad, \quad \epsilon=2 \sqrt{2}\left(2 \pi \alpha^{\prime}\right),
$$

and the corresponding tilded quantities

$$
\widetilde{\alpha^{\prime}}=\eta^{2} \alpha^{\prime}, \quad \widetilde{\epsilon}=\eta^{2} \epsilon .
$$

In particular, the constant $\epsilon$ is introduced to make contact with $[17,18]$. It plays the role of $\alpha^{\prime}$, but it is more convenient to use, since it absorbs the factor of $2 \pi$, which would otherwise appear explicitly in most of the equations that follow.

Let us now focus our attention on the description of brane states. In particular, let us start by concentrating on static BPS branes in $M-$ theory, given by embeddings $X^{\mu}(\xi)$ which satisfy $X^{ \pm}=\tau$ and $\dot{X}^{i}=0$, and which represent holomorphic maps of the manifold $\Sigma$ in the transverse space $X^{i}$ (as described in detail in [17]). Following the SenSeiberg limit described previously, we can consider the corresponding BPS brane states within the theory $\widetilde{M}$, or, better yet, within the corresponding Type IIA description. In particular, let us first introduce the rescaled coordinates on the world-volume of the brane

$$
\begin{aligned}
& \widetilde{\xi}^{\alpha} \sim\left(\widetilde{\tau}, \widetilde{\sigma}^{a}\right), \\
& \widetilde{\tau}=\tau, \quad \tilde{\sigma}^{a}=\eta^{2} \sigma^{a} .
\end{aligned}
$$

We are then considering, within the Type IIA description, a single static $D 2$-brane given by the embedding

$$
\begin{aligned}
\tilde{X}^{0}(\widetilde{\xi}) & =X^{+}(\xi)=\tau \\
\tilde{X}^{i}(\widetilde{\xi}) & =\eta^{2} X^{i}(\xi) .
\end{aligned}
$$

Moreover, since in the original $M$-theory description the light-cone momentum $P^{+}$of the brane is given by $P^{+}=2^{-1 / 2} P^{0}=2^{-1 / 2} T A(A$ 
denotes the area of the membrane), and since $P^{+}=N / R$, we must consider the sector of Type IIA with

$$
N=\frac{1}{\sqrt{2}} T A R
$$

units of $D 0$-brane charge.

As is well known [3], the motion of a D2-brane is described, in the low-energy limit, by the Born-Infeld (BI) action

$$
\widetilde{S}=-\widetilde{T} \int d^{3} \widetilde{\xi} \sqrt{-\operatorname{det}\left(\widetilde{g}_{\alpha \beta}+2 \pi \widetilde{\alpha}^{\prime} \widetilde{F}_{\alpha \beta}\right)},
$$

where the tension of the brane is given by

$$
\widetilde{T}=\frac{1}{(2 \pi)^{2} \widetilde{g_{s}} \widetilde{\ell}_{s}^{3}}=\eta^{-6} T
$$

and the induced metric by

$$
\widetilde{g}_{\alpha \beta}=-\widetilde{\partial}_{\alpha} \widetilde{X}^{0} \widetilde{\partial}_{\beta} \widetilde{X}^{0}+\widetilde{\partial}_{\alpha} \widetilde{X}^{i} \widetilde{\partial}_{\beta} \widetilde{X}^{i}
$$

This action, however, needs to be corrected at length scales comparable to the string length. These corrections take the form of terms with higher derivatives of the embedding functions and of the brane Maxwell field strength [23]. Nonetheless, for the case we are now considering of static BPS states, one may only consider the BI action, since higher derivative terms do not change the nature of the BPS solutions $[24,25]$. In particular, we have already seen that the embedding functions are given by a holomorphic map from $\Sigma$ to $X^{i}$. One then only needs to determine the correct Maxwell field on the membrane which describes the corresponding $M 2$-brane state. Recalling that $\widetilde{F}_{12}$ measures $D 0$ brane density on the $D 2$-brane, and using equation (4), we must have that

$$
\frac{1}{2 \pi} \int_{\Sigma} \widetilde{F}_{12} d^{2} \widetilde{\sigma}=\frac{\eta^{4}}{2 \pi} \int_{\Sigma} \widetilde{F}_{12} d^{2} \sigma=N=\frac{1}{\sqrt{2}} T A R
$$

It is not difficult to see that the correct solution to the BI action which satisfies the above normalization is given by

$$
\widetilde{F}_{12}=\frac{1}{\eta^{4} \sqrt{2}} \frac{Q}{2 \pi \alpha^{\prime}}
$$


where, as before, $Q d^{2} \sigma$ is the induced area element on the brane. We then have that for the static BPS case,

$$
2 \pi \widetilde{\alpha}^{\prime} \widetilde{F}_{\alpha \beta}=\frac{Q}{\eta^{2} \sqrt{2}}\left(\begin{array}{ccc}
0 & 0 & 0 \\
0 & 0 & 1 \\
0 & -1 & 0
\end{array}\right) .
$$

Let us now consider fluctuations around the static solution, within the Type IIA description. In order to make contact with the light-cone description of the $M 2$-brane, we shall use part of the reparametrization invariance of the BI action. In particular, we shall keep $\widetilde{X}^{0}=\widetilde{\tau}=$ $\tau$. Moreover, using time-dependent reparametrizations of the brane $\Sigma$ (which leave invariant the constraint $\widetilde{X}^{0}=\tau$ ), one can cancel any fluctuations $\widetilde{f}_{\alpha \beta}$ of the $U(1)$ field-strength $\widetilde{F}_{\alpha \beta}+\widetilde{f}_{\alpha \beta}$. This can be achieved by eliminating, first of all, the electric part $\tilde{f}_{0 a}$ with a $\tau$ dependent reparametrization, using the fact that we are working in a background with a large magnetic field (6). One can then use a $\tau-$ independent reparametrization to eliminate the magnetic part $\widetilde{f}_{12}$. This can be done since, in 2-dimensions, any two area elements (given in this case by $\widetilde{F}_{12} d^{2} \widetilde{\sigma}$ and by $\left.\left(\widetilde{F}_{12}+\widetilde{f}_{12}\right) d^{2} \widetilde{\sigma}\right)$ are always equivalent under reparametrization. Therefore, we can use diffeomorphism invariance on the world-volume to bring any given configuration of the D2-brane into a configuration with gauge field strength given by (6) and with $\widetilde{X}^{0}=\tau$.

One then only needs to consider fluctuations in the embedding functions. We consider generic transverse embedding functions

$$
\widetilde{X}^{i}(\widetilde{\xi})=\eta^{2} X^{i}(\xi)
$$

recalling that we are always looking at fluctuations which are finite from an M2-brane point of view, and are therefore finite in units of the Planck length. In units of the string length, these fluctuations are 
vanishingly small. The induced metric $\widetilde{g}_{\alpha \beta}$ is given by

$$
\begin{aligned}
\widetilde{g}_{\alpha \beta} & =\left(\begin{array}{cc}
-1+\widetilde{\partial}_{0} \widetilde{X}^{i} \widetilde{\partial}_{0} \widetilde{X}^{i} & \widetilde{\partial}_{0} \widetilde{X}^{i} \widetilde{\partial}_{b} \widetilde{X}^{i} \\
\widetilde{\partial}_{0} \widetilde{X}^{i} \widetilde{\partial}_{a} \widetilde{X}^{i} & \widetilde{\partial}_{a} \widetilde{X}^{i} \widetilde{\partial}_{b} \widetilde{X}^{i}
\end{array}\right) \\
& =\left(\begin{array}{cc}
-1+\eta^{4} \dot{X}^{i} \dot{X}^{i} & \eta^{2} \dot{X}^{i} \partial_{b} X^{i} \\
\eta^{2} \dot{X}^{i} \partial_{a} X^{i} & \text { partial }_{a} X^{i} \partial_{b} X^{i}
\end{array}\right) \\
& =\left(\begin{array}{cc}
-1+\eta^{4} \dot{X}^{i} \dot{X}^{i} & \eta^{2} \dot{X}^{i} \partial_{b} X^{i} \\
\eta^{2} \dot{X}^{i} \partial_{a} X^{i} & h_{a b}
\end{array}\right),
\end{aligned}
$$

where, as before, $h_{a b}=\partial_{a} X^{i} \partial_{b} X^{i}$ is the spatial part of the induced metric. We now wish to evaluate the BI action (5) in the presence of fluctuations. On general grounds one expects, in the limit of vanishingly small $\eta$, that

$$
\widetilde{S}=\int d \tau\left(-\frac{N}{\widetilde{R}}+L+o(\eta)\right)
$$

corresponding to the fact that the energies in Type IIA are related to the light-cone momenta in $M$-theory by $\widetilde{P}^{0}=N / \widetilde{R}+P^{+}$(Eq. 3 ). In the above equation, $L$ represents the Lagrangian in the $M$-theory limit, and we shall show that it corresponds to the Lagrangian (1). To compute the BI action, we introduce the short-hand notation

$$
M_{\alpha \beta}=\widetilde{g}_{\alpha \beta}+2 \pi \widetilde{\alpha}^{\prime} \widetilde{F}_{\alpha \beta} .
$$

Given the choice of gauge previously described, we have that

$$
M_{\alpha \beta}=\left(\begin{array}{ccc}
-1+\eta^{4} \dot{X}^{i} \dot{X}^{i} & \eta^{2} \dot{X}^{i} \partial_{1} X^{i} & \eta^{2} \dot{X}^{i} \partial_{2} X^{i} \\
\eta^{2} \dot{X}^{i} \partial_{1} X^{i} & h_{11} & \frac{Q}{\eta^{2} \sqrt{2}}+h_{12} \\
\eta^{2} \dot{X}^{i} \partial_{2} X^{i} & -\frac{Q}{\eta^{2} \sqrt{2}}+h_{21} & h_{22}
\end{array}\right)
$$

and, therefore,

$$
\begin{aligned}
-\operatorname{det} M_{\alpha \beta} & =\left(1-\eta^{4} \dot{X}^{i} \dot{X}^{i}\right)\left(\frac{Q^{2}}{2 \eta^{4}}+h\right)+o(\eta) \\
& =\frac{Q^{2}}{2 \eta^{4}}+h-\frac{Q^{2}}{2} \dot{X}^{i} \dot{X}^{i}+o(\eta),
\end{aligned}
$$


where $h=\operatorname{det} h_{a b}$. We can then evaluate the BI action in the $\eta \rightarrow 0$ limit (we drop, in the last lines, terms of order $\eta$ ),

$$
\begin{aligned}
\widetilde{S} & =-\widetilde{T} \int d^{3} \widetilde{\xi} \sqrt{-\operatorname{det} M_{\alpha \beta}} \\
& =-\frac{1}{\eta^{2}} T \int d^{3} \xi \sqrt{\frac{Q^{2}}{2 \eta^{4}}+h-\frac{Q^{2}}{2} \dot{X}^{i} \dot{X}^{i}+o(\eta)} \\
& =-\frac{1}{\eta^{4} \sqrt{2}} T \int d^{3} \xi Q\left(1+\frac{\eta^{4}}{Q^{2}} h-\frac{1}{2} \eta^{4} \dot{X}^{i} \dot{X}^{i}+o\left(\eta^{5}\right)\right) \\
& =\int d \tau\left(-\frac{T A}{\eta^{4} \sqrt{2}}+\frac{T}{\sqrt{2}} \int d^{2} \sigma\left(\frac{1}{2} Q \dot{X}^{i} \dot{X}^{i}-\frac{h}{Q}\right)\right) \\
& =\int d \widetilde{\tau}\left(-\frac{N}{\widetilde{R}}+L\right),
\end{aligned}
$$

where

$$
L=\frac{T}{\sqrt{2}} \int d^{2} \sigma\left(\frac{1}{2} Q \dot{X}^{i} \dot{X}^{i}-\frac{h}{Q}\right)
$$

Using the fact that

$$
h=\frac{1}{2} Q^{2}\left\{X^{i}, X^{j}\right\}^{2}
$$

we recover the Lagrangian (1).

To conclude this section we would like to derive, within the Type IIA description, the constraint (2) which is an integral part of the light-cone Lagrangian (1). Recall that we have used diffeomorphism invariance of the BI action to gauge away the fluctuations in the $U(1)$ field-strength. Invariance of the action $\widetilde{S}$ under infinitesimal fluctuations of $\widetilde{F}_{\alpha \beta}$ should then be reinterpreted as a constraint on the allowed configurations of the Lagrangian system under consideration. In particular, we shall show that invariance of $\widetilde{S}$ under infinitesimal fluctuations of the electric field will yield, in the $\eta \rightarrow 0$ limit, the constraint (2) on the embedding functions. Start by considering a generic fluctuation $\widetilde{f}_{\alpha \beta}=\partial_{\alpha} a_{\beta}-\partial_{\beta} a_{\alpha}$. Then

$$
\begin{aligned}
\widetilde{S}+\delta \widetilde{S} & \propto \int d^{3} \xi \sqrt{-\operatorname{det}\left(M_{\alpha \beta}+\widetilde{f}_{\alpha \beta}\right)} \\
\delta \widetilde{S} & \propto \int d^{3} \xi \sqrt{-\operatorname{det} M_{\alpha \beta}} M^{\alpha \beta} \widetilde{f}_{\beta \alpha}
\end{aligned}
$$


It is easy to see that, to leading order in $\eta$, the inverse matrix $M^{\alpha \beta}$ is given by (we only show the elements of the inverse matrix which will enter in the computation that follows)

$$
M^{\alpha \beta}=\frac{1}{\operatorname{det} M_{\alpha \beta}}\left(\begin{array}{ccc}
\cdots & -\frac{Q}{\sqrt{2}} \dot{X}^{i} \partial_{2} X^{i} & \frac{Q}{\sqrt{2}} \dot{X}^{i} \partial_{1} X^{i} \\
\frac{Q}{\sqrt{2}} \dot{X}^{i} \partial_{2} X^{i} & \ldots & \ldots \\
-\frac{Q}{\sqrt{2}} \dot{X}^{i} \partial_{1} X^{i} & \ldots & \ldots
\end{array}\right)+\cdots
$$

Let us now concentrate on the precise form of the infinitesimal fluctuation $\widetilde{f}_{\alpha \beta}$. First of all we can use $U(1)$ gauge invariance to move to the $a_{0}=0$ gauge. Moreover, since we are considering purely electric fluctuations, we have that $\partial_{1} a_{2}=\partial_{2} a_{1}$. Therefore $a_{a}=\partial_{a} f$. If we let $\lambda=\partial_{0} f$, we have that

$$
\tilde{f}_{0 a}=\partial_{a} \lambda \text {. }
$$

We can then use equation (7) to show that

$$
\begin{aligned}
\delta \widetilde{S} & \propto \int d^{3} \xi \frac{1}{\sqrt{-\operatorname{det} M_{\alpha \beta}}} \frac{Q}{\sqrt{2}}\left(\partial_{1} \lambda \dot{X}^{i} \partial_{2} X^{i}-\partial_{2} \lambda \dot{X}^{i} \partial_{1} X^{i}\right) \\
& \propto \int d^{3} \xi\left(\partial_{1} \lambda \dot{X}^{i} \partial_{2} X^{i}-\partial_{2} \lambda \dot{X}^{i} \partial_{1} X^{i}\right) \\
& \propto \int d^{3} \xi\left(\partial_{1} X^{i} \partial_{2} \dot{X}^{i}-\partial_{2} X^{i} \partial_{1} \dot{X}^{i}\right) \lambda .
\end{aligned}
$$

Since $\lambda$ is arbitrary, we recover the constraint,

$$
\partial_{1} X^{i} \partial_{2} \dot{X}^{i}-\partial_{2} X^{i} \partial_{1} \dot{X}^{i}=0=\left\{X^{i}, \dot{X}^{i}\right\},
$$

as given in equation (2).

\section{Higher Derivative Corrections to the Born-Infeld Action and the Star Prod- uct}

This final section is devoted to a qualitative discussion on the higherorder corrections to the Born-Infeld (BI) action and to the analysis of 
their Sen-Seiberg limit. The limit will be reinterpreted as a Matrix theory action living on the world-volume of the $M 2$-brane.

Let us start by recalling the Matrix theory Lagrangian $[6,8]$

$$
L=\frac{1}{R} \operatorname{Tr}\left(\frac{1}{2} \dot{X}^{i} \dot{X}^{i}+\frac{2}{\epsilon^{2}}\left[X^{i}, X^{j}\right]^{2}\right) .
$$

It is given by the dimensional reduction of 10-dimensional $U(N)$ SYMtheory to $(0+1)$-dimensions. It is the low-energy limit of the BI action describing the degrees of freedom of $N D 0$-branes and it has been conjectured $[6,8]$ to be an exact non-perturbative description of lightcone compactified $M$-theory, in the sector with $N$ units of momentum in the compact direction. The Lagrangian (8) should be supplemented with the constraint

$$
\left[X^{i}, \dot{X}^{i}\right]=0
$$

which comes from the choice of temporal gauge in the SYM theory. The conjecture itself can again be understood in terms of the Sen-Seiberg limit described in the previous section $[9,10]$.

The Lagrangian (8) should contain all of the physics of $M$-theory. In particular, it should describe sectors of the theory with finite $M 2$-brane charge. Let us consider, to start, a specific BPS M2-brane described at the classical level by a holomorphic curve in the transverse space. To be definite, introduce the complex coordinates $s=\sigma^{1}+i \sigma^{2}$ and $Z_{1}=X^{1}+i X^{2}, Z_{2}=X^{3}+i X^{4}, \ldots$, so that the coordinate functions $Z_{A}$ are analytic in $s$. It was shown in [17] that we can associate to each such curve a specific BPS state in Matrix theory, which gives a matrix representation of the given holomorphic brane. We shall not review here the construction given in [17], but we will quickly describe what is needed for the discussion in this section. In [17], given a fixed BPS brane, a map $\mathcal{Q}$ was constructed which associates to each function $A$ on the brane $\Sigma$ a matrix $\mathcal{Q}(A)$ (or better an operator on a given Hilbert space). The map $\mathcal{Q}$ has the property that, given any two functions $A$, $B$, on $\Sigma$, one has

$$
\mathcal{Q}(A) \mathcal{Q}(B)=\mathcal{Q}(A \star B)+o\left(\epsilon^{\infty}\right)
$$

where $\star$ is a specific associative star product associated with the given curve, which we shall describe in detail in what follows. As indicated 
above, the equality in equation (9) is asymptotic in $\epsilon$, and is valid only up to corrections which vanish faster then any power of $\epsilon$. In this paper we shall not be interested in non-perturbative effects in $\epsilon$ and will therefore ignore such corrections, treating the equality in (9) as exact. In this case, we can speak interchangeably of functions on the brane together with a given star product, or of matrices together with matrix multiplication. In order to make contact with the membrane theory of the last section, it is clear that the description in terms of functions on the brane is the preferable one, and we will, from now on, consider matrices as functions on $\Sigma$ (with the product $\star$ ).

Let us now describe star products in detail. A product $A \star B$ is given as a formal power series $\sum_{n=0}^{\infty} \epsilon^{n} S_{n}(A, B)$ in $\epsilon$, where at each order the coefficient $S_{n}(A, B)$ is a local bilinear in $A$ and $B$, built out of the derivatives of $A$ and $B$ up to finite order. In particular, we will have,

$$
\begin{aligned}
S_{0}(A, B) & =A B \\
S_{1}(A, B)-S_{1}(B, A) & =\frac{1}{2 i}\{A, B\} .
\end{aligned}
$$

As is well known [26, 27, 28, 29], the condition of associativity puts stringent constraints on the explicit form of the coefficients $S_{n}$.

We shall further constrain the star product by demanding that it preserves the BPS nature of the holomorphic brane which we are describing. In particular, the supersymmetry transformations of the Lagrangian (8) are known and simple, and one can show [17] that the correct BPS conditions which are appropriate for a supersymmetric membrane state are given by

$$
\begin{aligned}
& {\left[Z_{A}, Z_{B}\right]=\left[Z_{A}^{\dagger}, Z_{B}^{\dagger}\right]=0} \\
& \sum_{A}\left[Z_{A}, Z_{A}^{\dagger}\right]=-\epsilon .
\end{aligned}
$$

Imposing the above equations gives an extra constraint on the star product on the brane. It is carefully shown in [17] that the conditions above are solved by the following product

$$
A \star B=\sum_{n=0}^{\infty} \epsilon^{n} Q_{1} \cdots Q_{n}\left(\frac{1}{Q_{n}} \partial \cdots \frac{1}{Q_{1}} \partial A\right)\left(\frac{1}{Q_{n}} \bar{\partial} \cdots \frac{1}{Q_{1}} \bar{\partial} B\right) .
$$


The coefficients $Q_{n}$ are $(1,1)$ tensors on the brane, which can be computed recursively starting from $Q_{1}$ with the formula,

$$
Q_{n}=Q_{1}+Q_{n-1}+\epsilon \partial \bar{\partial} \log \left(Q_{1} \cdots Q_{n-1}\right) .
$$

The tensor $Q_{1}$ can, in turn, be computed perturbatively in $\epsilon$ by imposing equation (11) and is given by

$$
Q_{1}=-Q+\frac{\epsilon}{2} \partial \bar{\partial} \log Q+o\left(\epsilon^{2}\right)
$$

Let us also briefly discuss traces of operators. The major fact is that, again asymptotically in $\epsilon$, they can be computed as integrals over the surface $\Sigma$,

$$
\operatorname{Tr}(\mathcal{Q}(A))=\frac{1}{\epsilon} \int_{\Sigma} \sum_{n=0}^{\infty} \epsilon^{n} \mu_{n} A
$$

where

$$
\mu_{0}=\frac{1}{\pi \epsilon} Q d^{2} \sigma
$$

We now arrive to the fundamental point of this paper. One starts with a static BPS solution and considers the physics of the fluctuations, as governed by the Lagrangian (8). If one considers the fluctuations not as matrices but, in the spirit of what we have just discussed, as functions on the brane $\Sigma$, and if morover one replaces matrix products by star products and traces with integrals, one can rewrite the action (8) as a field theory on $\Sigma \times \mathbf{R}$ governing the fluctuations of the brane itself. Using equations $(10,12)$, we have

$$
\begin{aligned}
A \cdot B & \rightarrow A B+\cdots \\
{[,] } & \rightarrow \frac{\varepsilon}{2 i}\{,\}+\cdots \\
\operatorname{Tr} & \rightarrow \frac{1}{\pi \varepsilon} \int d^{2} \sigma Q+\cdots,
\end{aligned}
$$

Therefore the Matrix theory action (8) has an $\epsilon$ expansion which starts as

$$
L=\frac{1}{\pi \epsilon R} \int d^{2} \sigma Q\left(\frac{1}{2} \dot{X}^{i} \dot{X}^{i}-\frac{1}{2}\left\{X^{i}, X^{j}\right\}^{2}\right)+\cdots .
$$


The above reproduces the action (1), if one recalls that $\pi \epsilon R T=\sqrt{2}$. Moreover, the full gauge constraint $\left[\dot{X}^{i}, X^{i}\right]=0$ for the Matrix theory Lagrangian does imply, to leading order in $\epsilon$, the constraint $\left\{\dot{X}^{i}, X^{i}\right\}=$ 0 discussed in the last section.

Let us analyze higher order terms. From the point of view of the $D 2-$ brane action, one must understand the structure of the terms which involve higher derivatives of the embedding coordinates (curvature terms) and of the gauge field strength. These terms must be analyzed in the limit of very large background constant field-strength, as is clear from equation (6), even though we can work order by order in derivatives. Since we are working in the limit of vanishing string coupling, one can analyze open-string disk diagrams, where the underlying CFT is that of a string ending on a brane with a constant field-strength. Derivative corrections to the effective action can then be analyzed by computing standard string amplitudes, in the presence of the given background. At first sight we expect the derivative corrections to the BI action to diverge in the Sen-Seiberg limit. Indeed, we expect for example terms containing the curvature of the brane, measured in units of the string length. Since the characteristic length scale of the brane is proportional, in the Sen-Seiberg limit, to the Planck length, and since the string length $\widetilde{\ell}_{s} \sim \eta$ is large compared to $\widetilde{\ell}_{P} \sim \eta^{2}$, one expects curvature terms to dominate in the limit. However, in this argument we are neglecting the fact that we are working in a background of large gauge field strength $2 \pi \widetilde{\alpha}^{\prime} \widetilde{F}_{\alpha \beta} \sim 1 / \eta^{2}$. The curvature corrections can then be altered by inverse powers of the constant background gauge field strength (as can be seen by looking at the propagator in the boundary CFT in the presence of a background $F$ ), thus yielding a finite result.

It is therefore natural to conjecture the following. The Sen-Seiberg limit of the action describing the D2-brane in Type IIA string theory can be resummed to all orders in $\alpha^{\prime}$. The full sum can then be rewritten as the action (8) describing Matrix theory, where one interprets matrix multiplication as an associative star product on the brane worldvolume. The star product itself must, for consistency reasons, satisfy the conditions (10) and (11).

Let us conclude by commenting on some questions of uniqueness. In section 2 we carefully constructed the Sen-Seiberg limit by imposing the constraint (6). This clearly depends on the exact definition of 
the $U(1)$ gauge field living on the brane. In particular, recall that one can always do a field redefinition of the gauge field by adding gaugeinvariant quantities. Therefore, the precise form of the Sen-Seiberg limit depends on the chosen definition. It is then natural to expect that different definitions will yield in the limit actions written in terms of different star products. All these actions should be equivalent, since they can be resummed to yield the same Matrix theory action. Moreover, any consistent star product will have to be associative and will have to satisfy conditions (10) and (11).

\section{Acknowledgments}

We would like to thank W. Taylor for helpful discussions and comments, and also M. Douglas, A.A. Tseytlin and N. Seiberg for useful comments. One of us (RS) was supported in part by funds provided by the U.S. Department of Energy (D.O.E.) under cooperative research agreement $\sharp D E-F C 02-94 E R 40818$, in part by Fundação Calouste Gulbenkian (Portugal), and in part by Fundação Luso-Americana para o Desenvolvimento (Portugal).

\section{References}

[1] E. Witten, String Theory Dynamics in Various Dimensions, Nucl. Phys., B443 (1995), 85, hep-th/9503124.

[2] J. Polchinski, Dirichlet-Branes and Ramond-Ramond Charges, Phys. Rev. Lett., 75 (1995), 4724, hep-th/9510017.

[3] R. Leigh, Dirac-Born-Infeld Action from Dirichlet Sigma Model, Mod. Phys. Lett., A4 (1989), 2767.

[4] M. Douglas, D. Kabat, P. Pouliot, and S. Shenker, D-Branes and Short Distamces in String Theory, Nucl. Phys., B485 (1997), 85, hep-th/9608024.

[5] E. Witten, Bound States of Strings and p-Branes, Nucl. Phys., B460 (1996), 335, hep-th/9510135. 
[6] T. Banks, W. Fischler, S. Shenker, and L. Susskind, $M$-Theory as a Matrix Model: A Conjecture, Phys. Rev., D55 (1997), 5112, hep-th/9610043.

[7] T. Banks, N. Seiberg, and S. Shenker, Branes from Matrices, Nucl. Phys., B490 (1997), 91, hep-th/9612157.

[8] L. Susskind, Another Conjecture about M(atrix) Theory, hep-th/9704080.

[9] A. Sen, D0-Branes on $T^{n}$ and Matrix Theory, Adv. Theor. Math. Phys., 2 (1998), 51, hep-th/9709220.

[10] N. Seiberg, Why is the Matrix Model Correct?, Phys. Rev. Lett., 79 (1997), 3577, hep-th/9710009.

[11] C. Bachas, P. Bain, and M. Green, Curvature Terms in D-Branes Actions and their M-Theory Origin, JHEP, 9905 (1999), 011, hep-th/9903210.

[12] M. Douglas, D-Branes in Curved Space, hep-th/9703056.

[13] M. Douglas, A. Kato, and H. Ooguri, D-Brane Actions on Kähler Manifolds, hep-th/9708012.

[14] D. Kabat and W. Taylor IV, Linearized Supergravity from Matrix Theory, Phys. Lett., B426 (1998), 297, hep-th/9712185.

[15] W. Taylor IV and M. van Raamsdonk, Supergravity Currents and Linearized Interactions for Matrix Theory Configurations with Fermion Backgrounds, JHEP, 9904 (1999), 013, hep-th/9812239.

[16] W. Taylor IV and M. van Raamsdonk, Multiple D0-Branes in Weakly Curved Backgrounds, hep-th/9904095.

[17] L. Cornalba and W. Taylor IV, Holomorphic Curves from Matrices, Nucl. Phys., B536 (1998), 513, hep-th/9807060.

[18] L. Cornalba, Matrix Representations of Holomorphic Curves in $\mathrm{T}^{4}$, hep-th/9812184.

[19] N. Seiberg and E. Witten, Talks at the Strings '99 Conference, and private communications. 
[20] J. Goldstone, unpublished;

J. Hoppe, MIT, Ph.D. thesis (1982);

J. Hoppe, in proc. Int. Workshop on Constraint's Theory and Relativistic Dynamics; eds. G. Longhi and L. Lusanna (World Scientific, 1987).

[21] B. de Wit, J. Hoppe and H. Nicolai, On the Quantum Mechanics of Supermembranes, Nucl. Phys., B305 [FS 23] (1988), 545.

[22] J. Hoppe, Membranes And Integrable Systems, Phys. Lett., B250 (1990), 44.

[23] O.D. Anreev and A.A. Tseytlin, Partition-Function Representation for the Open Superstring Effective Action, Nucl. Phys., B311 (1988), 205.

[24] C. Callan and J. Maldacena, Brane Dynamics from the BornInfeld Action, Nucl. Phys., B513 (1998), 198, hep-th/9708147.

[25] G. Gibbons, Born-Infeld Particles and Dirichlet p-Branes, Nucl. Phys., B514 (1998), 603, hep-th/9709027.

[26] F. Bayen, M. Flato, C. Fronsdal, A. Lichnerowicz and D. Sternheimer, Deformation Theory and Quantization: Deformations of Symplectic Structures, Annals of Phys., 111 (1978), 61.

[27] M. de Wilde and P. Lecomte, Existence of Star Products and of Formal Deformations of the Poisson Lie Algebra of Arbitrary Symplectic Manifolds, Lett. Math. Phys., 7 (1983), 487.

[28] B. Fedosov, A Simple Geometrical Construction of Deformation Quantization, Jour. Diff. Geo., 40 (1994), 213.

[29] M. Kontsevich, Deformation Quantization of Poisson Manifolds I, q-alg/9709040. 